\title{
Improving the Quality of Bowel Preparation: Rewarding Patients for Success or Intensive Patient Education?
}

\author{
Marguerite J. Kutyla a, b Marcus A. Gray a, c, d Courtney von Hippel ${ }^{c}$ \\ Luke F. Hourigan ${ }^{a, e}$ Bradley J. Kendall ${ }^{a, e}$ Amanda J. Whaley ${ }^{a}$ \\ Sam O'Connor ${ }^{a}$ Gerald J. Holtmann ${ }^{a}, d, e, f$
}

${ }^{a}$ Department of Gastroenterology and Hepatology, Princess Alexandra Hospital, Woolloongabba, QLD, Australia; ${ }^{b}$ School of Pharmacy, Faculty of Health and Behavioural Sciences, University of Queensland, Brisbane, QLD, Australia; 'School of Psychology, Faculty of Health and Behavioural Sciences, University of Queensland, Brisbane, QLD, Australia; ${ }^{\mathrm{d}}$ Translational Research Institute, Brisbane, QLD, Australia; ${ }^{\text {EFaculty }}$ of Medicine, University of Queensland, Brisbane, QLD, Australia; ${ }^{f}$ Faculty of Health and Behavioural Sciences, University of Queensland, Brisbane, QLD, Australia

\section{Keywords}

Colonoscopy · Bowel preparation · Education · Adenoma · Incentive

\begin{abstract}
Introduction and Objectives: The quality of the bowel preparation is a critical parameter for the outcome of colonoscopies. It is well established that the bowel preparation modality (e.g., split or larger volume preparation) significantly improves the quality of the bowel preparation. Patient compliance is another important factor impacting on the quality of bowel preparations that receives relatively little research attention. We aimed to explore if intensified education or a lottery ticket as reward for good bowel preparation could improve outcomes. Methods: After informed consent, all patients received a standardized printed information booklet. In a randomized fashion, patients were offered (a) a lottery scratchy ticket with an opportunity to win $\$ 25,000$ as "reward" for good bowel preparation, (b) an education ses-
\end{abstract}

sion delivered over the phone by a trained nurse, or (c) no additional measure. Results: Overall, the quality of the bowel preparation was rated good or very good in $69.1 \%(95 \% \mathrm{Cl}$ $61.7-75.7 \%)$ of patients. Reward intervention did not influence the quality of bowel preparation (OR $0.42,95 \% \mathrm{Cl} 0.09$ $1.91, p=0.260)$; however, bowel preparation quality decreased in patients randomized to receive the additional education (OR 0.28, 95\% Cl 0.08-0.96, $p=0.042$ ). Neither intervention significantly impacted on polyp detection rates. Conclusions: Contrasting general beliefs, additional interventions (e.g., incentives or phone consultation) did not improve the quality of the bowel preparation. The unexpected result shows that utilizing extra resources must be balanced against real-world outcomes and may not always provide the expected result.

(c) 2020 S. Karger AG, Basel

Marguerite J. Kutyla and Marcus A. Gray contributed equally to this manuscript.

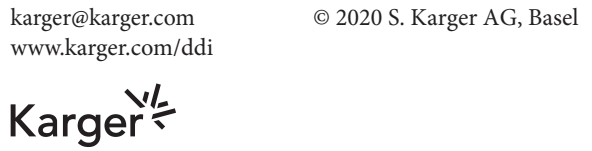

Gerald J. Holtmann

Department of Gastroenterology \& Hepatology, Princess Alexandra Hospital Brisbane University of Queensland

199 Ipswich Road, Woolloongabba QLD 4102 (Australia)

g.holtmann@uq.edu.au 


\section{Introduction}

Colonoscopy is a widely used diagnostic test that has been shown in the context of screening and surveillance to reduce colorectal cancer morbidity and mortality through early detection and removal of pre-cancerous lesions [1-3]. The quality of bowel preparation is a key determinant of the diagnostic yield of colonoscopies regarding polyp or adenoma detection rates, as polyp detection rate is adversely affected when bowel preparation is poor, due to decreased visualization [4-6]. It has been reported that up to one-third of all bowel preparations for colonoscopies are inadequate, and this translates into a requirement for repeat procedures or a reduced diagnostic yield [7-10]. In recent years, many interventions have aimed to improve the bowel preparation by modifying the regimen (e.g., split-preparation vs. single dose) and the ingredients (e.g., polyethylene glycol [PEG] vs. sodium phosphate solution) or to identify clinical risk factors such as underlying metabolic disorders [11-15]. However, very limited data are available in relation to measures that target patient compliance. In the setting of a publicly funded health service with free access to services, we aimed to determine if a potential financial reward (lottery ticket) or a one-to-one education session delivered over the phone would result in improved bowel cleansing outcomes for patients. Our secondary aim was to examine whether polyp or adenoma detection rates were influenced by the intervention.

\section{Materials and Methods}

This study was conducted at the Princess Alexandra Hospital (PAH), a tertiary/quaternary hospital affiliated with the University of Queensland, delivering $>12,000$ endoscopic procedures per year. After obtaining Human Research Ethics approval (Metro South Ethics Committee, HREC/15/QPAH/221), patients from the general gastroenterology clinic referred for an elective colonoscopy were approached by the clinical trials nurse and recruited into the study. Written informed consent was obtained from all participants. Exclusion criteria were significant cognitive or mental impairment, known need for interventional procedures (e.g., resection of large polyps), or language barriers. Block randomization was utilized to allocate patients into the 3 study arms, which was performed by one of the investigators (M.K.) the day following recruitment. The endoscopists who performed colonoscopies and assessed the quality of the bowel preparation were blinded to group allocation. The Boston Bowel Preparation Scale (BBPS) was used for assessment of colon cleansing where an overall score of $\geq 6$ and each individual segment $\geq 2$ considered as adequate preparation [16]. Our primary measure of overall Bowel Preparation Quality (we term this score $\mathrm{BPQ})(0-3$, where 0 = poor, 1 = fair, 2 = good, and 3 = excellent) was set as the lowest BBPS score across any section of the bowel. Our sample size was determined from an independent but representative sample of the 4,321 patients who had undergone colonoscopies within our department from January to November of 2018. Of these, 4,097 had BPQ data (mean $1.80 \pm$ 0.705 ). For an $\alpha=0.05$ and a $\beta=0.8$, our study allows detection of a mean group change of 0.18 points (BPQ) or larger in either direction $(<1.62$ or $>1.98)$. This equates to an effect size of 0.256 in each direction (2-tailed). An allocation ratio of 2.2 requires 44 patients in the control group and 98 patients in the combined intervention groups. These sample sizes were met.

The first group received standard care. All patients were prescribed a 3-L split-dose PEG bowel preparation and a 12-page colour information booklet was mailed to the participants a month prior to the procedure (see online suppl. material; see www.karger.com/doi/10.1159/000510461 for all online suppl. material). The text of the information booklet required a grade 6 level of education (standard). The second group received standard care plus they were offered a financial reward (a lottery ticket for a state-wide lottery with a jackpot of up to $\$ 25,000$ ) if they achieved a "good or very good" quality rating of the preparation. The third group received standard care plus additional one-on-one education 2 weeks prior to their procedure, delivered by an endoscopy nurse over the phone. The education consisted of a brief run-down of the instructions in the booklet, possible adverse events that could be experienced, and emphasis on the importance of following the instructions to attain adequate bowel cleansing. Conversations usually lasted between 5 and 30 min, depending on the interaction. Fourteen education patients required more than one phone contact attempt, with nursing staff attempting daily contact and leaving voice mail messages (if available) requesting a return call. Two patients were not able to be contacted at all.

Demographic information collected included age, gender, employment, and relationship status. Clinical information included the presence of significant comorbidities, indicated by any of the following: cardiovascular disease, obesity, renal impairment, hepatic impairment, asthma or chronic obstructive pulmonary disease, diabetes mellitus, brain injury, inflammatory bowel disease, previous or current cancer, hypothyroidism, or chronic pain. Finally, data recorded during colonoscopy included quality of bowel preparation (BBPS) and the presence of adenomas or polyps.

\section{Statistical Analysis}

Comparisons of groups (age, gender, relationship status, and presence of significant medical comorbidities) were done with independent samples $t$ tests, and associations between confounds examined with Pearson's bivariate correlations. Group differences in indicators for colonoscopy were examined with $\chi^{2}$ analysis. The primary aim (influence of intervention on bowel preparation quality) was examined with binary logistic regression after dichotomizing bowel preparation for excellent or good preparation versus fair or poor preparation outcomes. Likewise, influence of bowel preparation on the detection of adenomas or polyps was examined via logistic regression. Logistic regression analyses controlled for group differences by including age and comorbidities as covariates of no interest. 


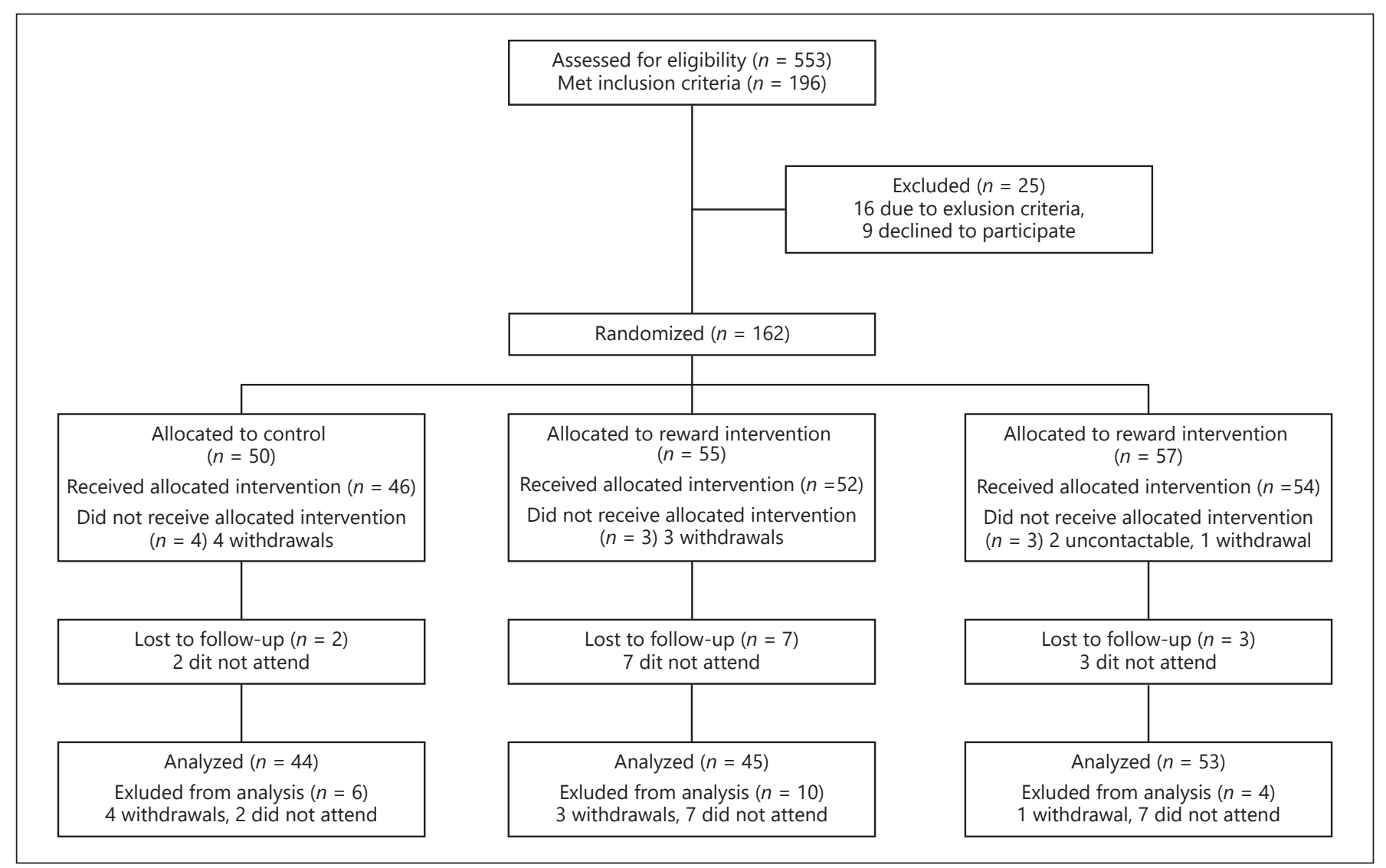

Fig. 1. Study randomization and analysis diagram.

Table 1. Patient socio-demographic details, clinical factors, and intervention outcomes

\begin{tabular}{|c|c|c|c|c|}
\hline Mean age $\pm S D[95 \% \mathrm{CI}]$ & $43.1 \pm 16[38-48]$ & $55.0 \pm 17[50-60]^{\mathrm{a}}$ & $48.3 \pm 16[44-53]$ & $51.1 \pm 16[48-54]^{\mathrm{b}}$ \\
\hline Male gender $[95 \% \mathrm{CI}]$ & $20(45 \%)[30-61 \%]$ & $20(44 \%)$ [29-60\%] & $24(45 \%)[31-61 \%]$ & $45(45 \%)[35-55 \%]$ \\
\hline In a relationship $[95 \% \mathrm{CI}]$ & $27(61 \%)[46-76 \%]$ & $23(51 \%)[36-66 \%]$ & $24(45 \%)[31-59 \%]$ & $48(48 \%)[34-54 \%]$ \\
\hline Significant comorbidities $[95 \% \mathrm{CI}]$ & $4(9 \%)[2.5-18 \%]$ & $20(44 \%)[29-60]^{\mathrm{a}}$ & $15(28 \%)[16-41 \%]^{\mathrm{b}}$ & $35(36 \%)[26-45 \%]^{\mathrm{a}}$ \\
\hline Adenoma detection rates $[95 \% \mathrm{CI}]$ & $12(27 \%)[14-41 \%]$ & $22(49 \%)[34-64 \%]^{\mathrm{b}}$ & $24(45 \%)[31-59 \%]^{\mathrm{c}}$ & $46(46 \%)[36-56 \%]^{\mathrm{b}}$ \\
\hline Polyp detection rates $[95 \% \mathrm{CI}]$ & $19(43 \%)[28-58 \%]$ & $26(58 \%)[43-73 \%]^{\mathrm{d}}$ & $31(58 \%)[45-72 \%]^{\mathrm{d}}$ & $58(59 \%)[49-68 \%]^{\mathrm{d}}$ \\
\hline
\end{tabular}

These group comparisons do not correct for confounds. ${ }^{a}$ Significantly different from control, $\mathrm{p}<0.005$. ${ }^{\mathrm{b}}$ Significantly different from control, $p<0.05$. $^{\mathrm{c}}$ Trend for difference from control, $p<0.06 .{ }^{\mathrm{d}}$ No difference from control, $p \geq 0.099$.

\section{Results}

\section{Comparison of Intervention Groups}

The characteristics of the various groups are shown in Table 1 . There were no statistically significant differences regarding gender distribution and relationship status. Patients in the intervention groups were older than controls (Table 1).

Two patients of the 50 controls, 7 patients of 55 in the incentive group, and 3 patients of 57 in the education 


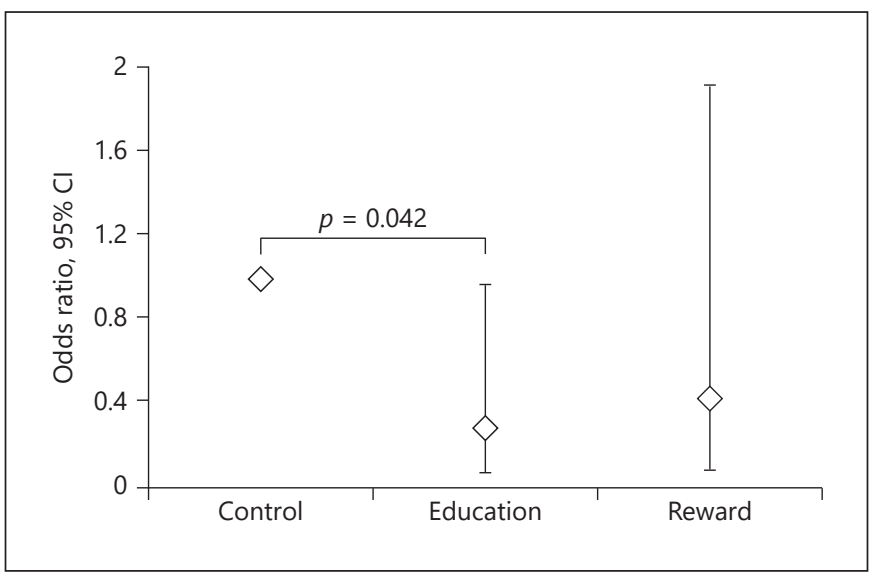

Fig. 2. Odds ratios of good or very good bowel preparation score after standard (written) information, after a dedicated 30-min education session, and with a reward $( \pm 95 \% \mathrm{CI})$.

group did not attend their procedure. Thus, data on the quality of the bowel preparation was not available for these patients. Likewise, 4 patients from the control group, 3 from the reward group, and 1 from the education group withdrew their consent to participate in the study; hence, their results were excluded (Fig. 1).

\section{Effect of Interventions on the Quality of Bowel \\ Preparation}

In control patients, 40 (91\%) achieved a good or very good quality of bowel preparation. In patients who were offered the lottery ticket reward for good or better quality of bowel preparation, 34 (76\%) achieved the desired result. In the education intervention patients, good or better preparation was achieved in 38 patients (72\%). Therefore, additional interventions did not improve the bowel preparation quality. Moreover, even when correcting for confounds, the additional education intervention significantly reduced bowel preparation quality (OR 0.28, 95\% CI 0.08-0.96, $p=0.042$ ), whereas the impact of the reward intervention on the quality of bowel preparation was reduced to a non-significant trend (OR $0.42,95 \%$ CI $0.09-1.91, p=0.260$ ) (Fig. 2). Similarly, when considered together, the interventions did not influence the quality of bowel preparation (OR 0.350, 95\% CI $0.11-1.1, p=0.074$ ).

\section{Effect of Interventions on Adenoma Detection Rates}

In the control group, 12 patients (27\%) had adenomas detected, whereas in the reward and education groups, 22 (49\%) and $24(45 \%)$ patients had adenomas. Age is of considerable importance as the incidence of features identified during colonoscopy typically increases with patient age. This was also true in our patients: those with adenomas were on average 10 years older ( 54.5 vs. 44.6 years), with each year increasing risk of adenoma by $4 \%$, (OR 1.039, 95\% CI 1.016-1.062, $p=0.001$ ). Correcting for the confounders, the interventions did not significantly influence the detection rates for adenomas.

\section{Discussion/Conclusion}

Patients undergoing bowel preparation were randomly assigned to the 3 different study arms. While requiring additional resources, the 2 interventions (incentive and intensified education) did not result in improved bowel preparation outcomes compared to the control arm. This result highlights the importance of balancing increased healthcare resources against real-world outcomes.

A review by Jochelson [17] concluded that incentive schemes were most successful when aimed at influencing simple, short-term actions of the patient. Since compliance with bowel preparation is a short-term behavioural change, rewarding positive patient behaviour could be considered a potential approach to improve the quality of bowel preparation. However, contrasting our expectations, this intervention resulted in a negative, although non-significant, outcome for the quality of preparation. Nevertheless, the lack of positive impact is an interesting observation. The possibility of winning a substantial amount of money $(\$ 25,000)$ was offset by the slim likelihood of winning ( 1 in 825,000) although most patients were unlikely to be aware of the actual odds. However, it is possible that patients would likely view the reward at the $\$ 2$ face value (the cost of the lottery ticket), rather than the $\$ 25,000$ jackpot prize. We surmise that patients considered the trade-off of missing out on the reward as small, compared to the immediate task of drinking litres of unpleasant bowel preparation. Hence, the "real" value was too small to motivate the subset of poorly compliant patients to adhere to their bowel preparation instructions. Indeed, incentives, even for relatively simple tasks such as stool testing for faecal occult blood (FOBT), have been shown to be largely ineffective, with similar rates of screening uptake between no incentive versus a $\$ 5$ or $\$ 10$ reward [18]. Another incentive study also showed similar results, whereby a reward of up to $\$ 20$ showed no change on FOBT screening rates [19]. Interestingly, it also showed that an entry into a raffle worth $\$ 500$ resulted in no change to uptake 
(chances of winning were approximately 1 in 100) but a 1 -in-10 chance of receiving $\$ 50$ proved effective in improving screening rates. This indicates that there is an interaction between the value of the financial reward and the perceived certainty of obtaining it. There is also the possibility that an extrinsic motivator (reward) can reduce patients' intrinsic motivation and have the opposite effect to the one intended [20]. A common demographic at the study site are patients from low SES backgrounds who possess low health literacy and/or low health engagement; thus, the small reward could have further obscured the "real" cost of non-compliance, such as missed adenomas, increased complications, and longer procedure times as a consequence of poor preparation. These patients, however, would be expected to benefit from intensified education. This is where the second intervention (intensified education via phone counselling) would be more appropriate.

Patients in the intensified education arm of the study received phone counselling by an endoscopy nurse a fortnight prior to their scheduled procedure, in addition to a standardized booklet that was supplied to all patients. This intervention was expected to increase patients' understanding of what was required for a successful bowel preparation and why it was important to prepare well, as well as presented them with an opportunity to ask questions and clarify any concerns. It was surprising, therefore, to find that this intervention worsened patient outcomes with regard to quality of preparation (Table 1). This seems to contrast the current literature where a recent systematic review has indicated that extra education is of benefit [21]. Two other meta-analyses that posed the same question concluded that enhanced instructions (e.g., cartoons and smartphone apps) are likely to improve bowel preparation quality, but the varied purgatives used in the studies reviewed warranted further investigation and the analyses were limited due to the identified publication bias in the studies [22, 23].

While the tested measures did not result in an improvement of the quality of bowel preparations, it could be argued that standard care (that included split preparation and a simplified, easy-to-read, instructional booklet with pictorial guidance) caused a "ceiling effect." Indeed, this is corroborated by Lee et al. [24, 25] who showed a significant improvement in preparation quality in patients on single-dose preparation after developing a novel education booklet versus usual instructions, but this improvement effect was lost when the booklet was tested in a study with split-preparation cohorts with a higher baseline. Whilst giving the patients information is imperative for their understanding and engagement, too much information, or information overload, may have caused them to feel overwhelmed. Nonetheless, the elements for information overload in the healthcare setting include low patient education level, health literacy, and socioeconomic status [26], which are the same factors that place such patients in need of extra education. The results of our study present an opportunity to review the education methods used and tailor them towards such patients.

In summary, this randomized controlled trial reveals that in outpatients undergoing elective colonoscopy, simple, written education material yields high rates of good bowel preparation. Additional education via the phone or an incentive to improve the quality of the bowel preparation does not result in better outcomes.

\section{Statement of Ethics}

Ethics approval for the study was obtained from the Metro South Ethics Committee (HREC/15/QPAH/221). Written informed consent was obtained from all participants recruited into the study.

\section{Conflict of Interest Statement}

The authors have no conflicts of interest to declare.

\section{Funding Sources}

This project was funded by the Princess Alexandra Hospital Research Support Scheme, 2015.

\section{Author Contributions}

M.K., C.V., A.W., and G.H. were involved in planning the original project details; M.K., M.G., C.V., L.H., B.K., A.W., S.O., and G.H. provided intellectual input and played a role in implementation of the project. M.K. was involved with data collection; M.K., M.G., C.V., and G.H. played a role in analyzing and interpreting data; M.K., M.G., and G.H. played a role in drafting the manuscript; M.K., M.G., C.V., L.H., B.K., A.W., S.O., and G.H. played a role in reviewing and revising the manuscript for important intellectual content. All authors approved the final version of the manuscript. 


\section{References}

1 Kahi CJ, Anderson JC, Rex DK. Screening and surveillance for colorectal cancer: state of the art. Gastrointest Endosc. 2013;77(3):335-50.

2 Lieberman D. Screening, surveillance, and prevention of colorectal cancer. Gastrointest Endosc Clin N Am. 2008;18(3):595-605,xi.

3 Winawer SJ, Zauber AG, O'Brien MJ, Ho MN, Gottlieb L, Sternberg SS, et al. Randomized comparison of surveillance intervals after colonoscopic removal of newly diagnosed adenomatous polyps. The National Polyp Study Workgroup. N Engl J Med. 1993;328(13): 901-6.

4 Chokshi RV, Hovis CE, Hollander T, Early DS, Wang JS. Prevalence of missed adenomas in patients with inadequate bowel preparation on screening colonoscopy. Gastrointest Endosc. 2012;75(6):1197-203.

5 Basch CH, Hillyer GC, Basch CE, Lebwohl B, Neugut AI. Characteristics associated with suboptimal bowel preparation prior to colonoscopy: results of a national survey. Int J Prev Med. 2014;5(2):233.

6 Lebwohl B, Kastrinos F, Glick M, Rosenbaum AJ, Wang T, Neugut AI. The impact of suboptimal bowel preparation on adenoma miss rates and the factors associated with early repeat colonoscopy. Gastrointest Endosc. 2011; 73(6):1207-14

7 Hillyer GC, Basch CH, Lebwohl B, Basch CE, Kastrinos F, Insel BJ, et al. Shortened surveillance intervals following suboptimal bowel preparation for colonoscopy: results of a national survey. Int J Colorectal Dis. 2013;28(1): 73-81.

8 Rex DK, Imperiale TF, Latinovich DR, Bratcher LL. Impact of bowel preparation on efficiency and cost of colonoscopy. Am J Gastroenterol. 2002;97(7):1696-700.

9 Hassan C, Fuccio L, Bruno M, Pagano N, Spada C, Carrara S, et al. A predictive model identifies patients most likely to have inadequate bowel preparation for colonoscopy. Clin Gastroenterol Hepatol. 2012;10(5):501.

10 Kazarian ES, Carreira FS, Toribara NW, Denberg TD. Colonoscopy completion in a large safety net health care system. Clin Gastroenterol Hepatol. 2008;6(4):438-42.
11 Adams WJ, Meagher AP, Lubowski DZ, King DW. Bisacodyl reduces the volume of polyethylene glycol solution required for bowel preparation. Dis Colon Rectum. 1994;37(3): 229-4.

12 Cheng RW, Chiu YC, Wu KL, Rayner CK, Tai WC, Hu ML, et al. Predictive factors for inadequate colon preparation before colonoscopy. Tech Coloproctol. 2015;19(2):111-5.

13 Romero RV, Mahadeva S. Factors influencing quality of bowel preparation for colonoscopy. World J Gastrointest Endosc. 2013;5(2):39-46.

14 Park SS, Sinn DH, Kim YH, Lim YJ, Sun Y, Lee JH, et al. Efficacy and tolerability of splitdose magnesium citrate: low-volume (2 liters) polyethylene glycol vs. single- or split-dose polyethylene glycol bowel preparation for morning colonoscopy. Am J Gastroenterol. 2010;105(6):1319-26.

15 Wexner SD, Beck DE, Baron TH, Wexner SD, Beck DE, Baron TH, et al. A consensus document on bowel preparation before colonoscopy: prepared by a task force from the American Society of Colon and Rectal Surgeons (ASCRS), the American Society for Gastrointestinal Endoscopy (ASGE), and the Society of American Gastrointestinal and Endoscopic Surgeons (SAGES). Surg Endosc. 2006;20(7): 1147-60.

16 Lai EJ, Calderwood AH, Doros G, Fix OK, Jacobson BC. The Boston bowel preparation scale: a valid and reliable instrument for colonoscopy-oriented research. Gastrointest Endosc. 2009;69(3 Pt 2):620-5.

17 Jochelson K. Paying the patient: improving health using financial incentives. London: King's Fund; 2007.

18 Gupta S, Miller S, Koch M, Berry E, Anderson $\mathrm{P}$, Pruitt SL, et al. Financial incentives for promoting colorectal cancer screening: a randomized, comparative effectiveness trial. Am J Gastroenterol. 2016;111(11):1630. : https:// www.nature.com/articles/ajg2016286\# supplementary-information.http: //dx.doi. org/10.1038/ajg.2016.286.
19 Kullgren JT, Dicks TN, Fu X, Richardson D, Tzanis GL, Tobi M, et al. Financial incentives for completion of fecal occult blood tests among veterans: a 2-stage, pragmatic, cluster, randomized, controlled trial. Ann Intern Med. 2014;161(10 Suppl):S35-43.

20 Gneezy U, Meier S, Rey-Biel P. When and why incentives (don't) work to modify behavior. J Econ Perspect. 2011;25(4):191-210.

21 Kurlander JE, Sondhi AR, Waljee AK, Menees SB, Connell CM, Schoenfeld PS, et al. How efficacious are patient education interventions to improve bowel preparation for colonoscopy? A systematic review. PLoS One. 2016;11(10):e0164442.

22 Guo X, Yang Z, Zhao L, Leung F, Luo H, Kang $X$, et al. Enhanced instructions improve the quality of bowel preparation for colonoscopy: a meta-analysis of randomized controlled trials. Gastrointest Endosc. 2017;85(1):90.

23 Chang CW, Shih SC, Wang HY, Chu CH, Wang TE, Hung CY, et al. Meta-analysis: the effect of patient education on bowel preparation for colonoscopy. Endosc Int Open. 2015; 3(6):E646-52.

24 Spiegel BM, Talley J, Shekelle P, Agarwal N, Snyder B, Bolus R, et al. Development and validation of a novel patient educational booklet to enhance colonoscopy preparation. Am J Gastroenterol. 2011;106(5):87583. http://www.nature.com/ajg/journal/v106/ n5/suppinfo/ajg201175s1.html.http://dx.doi. org/10.1038/ajg.2011.75.

25 Lee A, Vu M, Fisher DA, Modi R, Baek MD, Johnson MR, et al. Further validation of a novel patient educational booklet to enhance colonoscopy preparation: benefits in singledose, but not splitdose preparations. Gastroenterology. 2013;144:S191.

26 Khaleel I, Wimmer BC, Peterson GM, Zaidi STR, Roehrer E, Cummings E, et al. Health information overload among health consumers: a scoping review. Patient Educ Couns. 2020;103(1):15-32. 